\title{
A Survey and Qualitative Analysis of Multi-channel MAC Protocols for VANET
}

\author{
Jagadeesh Kakarla \\ Student \\ Department of Computer Science, \\ Pondicherry University, \\ Pondicherry-India
}

\author{
S Siva Sathya \\ Associate Professor \\ Department of Computer Science, \\ Pondicherry University, \\ Pondicherry-India
}

\begin{abstract}
Vehicular Ad-hoc Network (VANET) is an emerging and promising technology that places great demand on the access capability of the existing wireless technology. In VANET one primary issue is Medium Access Control (MAC), which aims to utilize the radio spectrum efficiently, to resolve potential contention and collision among vehicles for using the medium. Contention reduces the performance of single channel MAC layer and the workload distribution is also not well defined. So multi channel MAC protocols are useful to provide better quality of services (QoS) wherein, multi channel interference is a major problem to assign channel to a particular vehicle. Hence a survey is necessary on multi channel MAC protocols based on various parameters in vehicle to vehicle (V2V) and vehicle to infrastructure (V2I) communication. This paper gives a brief overview of various issues involved in the design of multi channel MAC protocol in VANET.
\end{abstract}

\section{Keywords}

Control channel, Service channel, Beacon period, Coordinating Access Point, Beam Table, Adaptive Broadcast Frame and Road side unit.

\section{INTRODUCTION}

Vehicular Ad hoc Network (VANET) is a peculiar type of Mobile Ad hoc Network (MANET). In VANET [21] each vehicle acts as a router to exchange data between nodes in the network. It is designed for vehicle-to-vehicle (V2V) and infrastructure-to-vehicle (IVC) communication. Such networks are used in traffic control applications, safety applications, driver assistance and location based services. VANET has unique characteristics like high mobility with the constraint of road topology, initially low market penetration ratio, unbounded network size, infrastructure support that differentiate it from MANET.

Vehicular ad hoc networks (VANETs) have been considered as an important communication infrastructure for the Intelligent Transportation Systems (ITS). Based on IEEE 802.11p [1], VANET uses Dedicated Short Range Communication (DSRC) for the enrichment of drivers comfort and safety. The U.S.Federal Communication Commission (FCC) has allocated $75 \mathrm{MHz}$ of spectrum at $5.9 \mathrm{GHz}$ exclusively for vehicular communications. The overall spectrum is divided into seven $10 \mathrm{MHz}$ channels [12]. The Channel 178 is assigned as Control Channel $(\mathrm{CCH})$ for the transmission of announcements and short data messages. The other channels are designated as Service Channels (SCHs), where additional data transfers and special operations applications. Channel 172 and Channel 184 are used for safety application in $\mathrm{V} 2 \mathrm{~V}$ communication. It allows a data payload communication capability of $3,4.5,6,9,12,18,24$, and 27 Mbps using the optional $20 \mathrm{MHz}$ channels, it allows data payload capabilities up to 54 Mbps. Many single-channel [14] [19] [6] MAC (medium access control) protocols that have been developed for VANET, all vehicles share a common channel in the network. Wireless Access in Vehicular Environments (WAVE) MAC uses contention-based access using Carrier Sense Multiple Access with Collision Avoidance (CSMA/CA) mechanism. Due to high contentions and collisions, the performance of single-channel MAC protocols degrades quickly with the increase in density of vehicles. Menouar [11] also concluded that the IEEE 802.11 MAC protocol is not suitable for real-time traffic and QoS provision. So multi channel MAC protocols are useful to provide better quality of services (QoS).

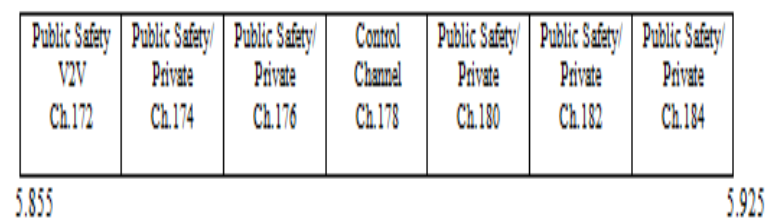

Fig 1: 5.9 GHz DSRC spectrum

The rest of the paper is organized as follows. Section 2 discusses related work, section 3 deals with various Multichannel MAC Protocols both for Adhoc networks and VANETS and section 4 concludes the paper.

\section{RELATED WORK}

The need for developing protocols which could overcome the collisions resulting from single channel MAC was felt and initially two different schemes were put forth. They are Distributed and Centralized Adhoc multichannel coordination schemes. These protocols paved the way for the development of the existing Multi-channel MAC protocols.

\subsection{Distributed Ad-hoc Multi-Channel Coordination Schemes:}

Yunpeng Zang [2] proposed a novel MAC protocol called as Vehicular MESH Network (VMESH) which is a compliant of WAVE multi-channel operation system and based on a distributed beaconing scheme. It divides the control channel $(\mathrm{CCH})$ into Beacon Period (BP) and the Safety Period (SP). In each Beacon period all vehicles should transmit a beacon packet which contains information for making dynamic resource reservation on service channels $(\mathrm{SCH})$. It provides contention free access on SCHs to improve the throughput of 
non-safety applications. This protocol uses IEEE 802.11 DCF as the channel access mechanism. Broadcast message from a vehicle suffers collisions from neighbors which are accessing the channel. This protocol dynamically adjusts the $\mathrm{CCH}$ based on density of vehicles to offer supports for safety applications and limits the available share for non-safety applications by the long $\mathrm{CCH}$ interval.

Jiang et al. [5] presents a Peercast protocol. Every vehicle proactively echoes a received safety message from its neighbors. If a vehicle does not hear either any safety message or echo message on the control channel then it can switch to the service channel to transfer non-safety messages. This protocol improves the performance of non-safety applications, but it cannot give guarantee for receiving emergency warning messages, when a vehicle is operating in a service channel.

\subsection{Centralized Multi-Channel Coordination Schemes:}

Tony K. Mak [10] proposed a centralized MAC protocol called as Dedicated Coordinating Access Point (DCAP) to enable multichannel operation for dedicated short range communications (DSRCs). Each DCAP contains a Coordinating Access Point (CAP) and one or more Service Access Points (SAP) to provide non-safety applications in the region. DCAP divides time into periodic regulated intervals, called the repetition period. The length of repetition period is determined by the maximum tolerable delay of safety messages. Each repetition period is divided into two distinct sub periods: contention free period (CFP) and contention period (CP). In CFP, DCAP sends a broadcast packet to access the channel and polls each vehicle individually to transmit its safety messages, where remaining vehicles must remain silent. The nodes that are not polled in the CFP will contend the channel in the following CP. This protocol permits vehicles to transmit only one safety message per CFP. DCAP avoids channel interference during the CFP by partitioning the communication range of control channel radio into multiple different radius circular regions with a center at the CAP.

\section{MULTI-CHANNEL MAC PROTOCOLS}

The concept of multi-channel MAC Protocols were initially developed only for Adhoc networks and only later its need was felt for VANET's also. Hence this section focuses on multi-channel MAC Protocols for both the categories.

\subsection{Multi-channel MAC Protocols for Adhoc Networks}

Nakjung Choi [17] proposed a Multi-Channel MAC Protocol for Mobile Ad Hoc Networks to increase overall network performance, reliability and provided a solution for the Hidden Multi-Channel Problem. Among the multiple channels, one channel is reserved for control messages, and the remaining channels are available for data transfer. By observing the control channel, a node can get the status of data channels. Control channel is used to transfer control messages, while data is transferred through the data channel. In negotiation of data channel CONFIRM message is added to RTS, CTS and ACK messages. CONFIRM message indicates which data channel is used for the communication. Every node maintains a channel table that contains information about all data channels. The channel table contains the Maximum Transmission Time which is defined for each data channel i.e. is the maximum time allowed for a packet to be transmitted over the particular channel and Channel Allocation Vector (CAV) is associated for each channel to indicate the time reserved for data transmission over the channel. Initially $\mathrm{CAV}$ value is declared as packet length contained in the control messages over the channel, and is decremented at each time unit.

DYNAMMA: Venkatesh [20] introduced A Dynamic Multichannel Medium Access Framework for Wireless Ad Hoc Networks. In this protocol the channel access time is partitioned into superframes. Each superframe consists of a fixed number of time slots and these slots are of three different types: signaling slots, base data slots and burst data slots. Signaling slots are used to gather information about neighbors and traffic, while base data slots and burst data slots used for data exchange. The length of a signaling slot is based on the maximum signaling frame duration, base data slots and burst data slots are based on the physical layer transmission rate, data packet size, number of data packets to be transmitted within the burst, channel switching time, and radio turn-on time. The superframe structure provides support for neighbor discovery and traffic adaptation.

Jonathan Wellons [4] developed a performance-ratio robust routing protocol for multi-radio, multi-channel wireless sensor networks. The author has proposed a Protocol Model where packet transmission from node $\mathrm{u}$ to $\mathrm{v}$ is successful if the two nodes are in transmission range with each other or any other node within the range of two nodes is not sending data on the same channel. The performance [16] of any multi-radio multichannel protocol in wireless mesh network mainly depends on scheduling, channel assignment, and routing algorithms. So in this protocol, the author derived a routing solution based on the necessary conditions of channel assignment and schedulability to provide a feasible solution.

SNMS: Sheng-Hsuan Hsu [7] introduced Multi-Channel MAC Protocol based on Sequenced Neighbor with Multicast Support. This protocol uses Sender-based data transmission scheme to provide efficient multicast and uses one control channel for transmission of control packets and several data channels to deliver data packets. In Neighbor-Sequenced method, a node can identify its neighbor set and assign a unique sequence number for them. Whenever a node moves out of the region, then it will send a DELETE packet with its sequence number to all its neighbors. After receiving DELETE packet, the neighbors will check whether the sequence number is less than its own sequence number. If it is YES, then neighbors of the node will decrease its sequence number by one. Each node maintains neighbor table that contains sequence numbers and channel numbers of its neighbors. Later Zhijun Cai [13] proposed a Sequenced Neighbor Double Reservation Multi-channel Ad Hoc Networks based on receiver-based data transmission scheme, similar to SNMS protocol. Nessrine Chakchouk [9] proposed Multiradio Multichannel Traffic and Interference Aware Scheduling algorithm. The protocol increases the throughput of the network by eliminating the interference between the routers.

CAM-MAC: Tie Luo [12] proposed a Cooperative Asynchronous Multi-Channel MAC Protocol for Ad Hoc Networks, which is a simple protocol to implement than other multi-channel MAC protocols. The author introduced cooperation into multi-channel ad hoc networks to gain the knowledge of channel conditions, which improves the overall performance. Suppose a communication is to be established 
between source and destination nodes, and no sufficient information is available to select collision-free channel, then nodes can gather information from neighbor nodes, rather than selecting channels independently.

Due to unique characteristics like high mobility with the constraint of road topology, initially low market penetration ratio, unbounded network size, infrastructure support for VANET differentiates it from other Adhoc networks. Hence all the above described Multi-channel MAC Protocols does not give better performance in vehicular environment.

\subsection{Multi-channel MAC Protocols for VANET}

Currently there are several Multi-channel MAC protocols for VANET which are proved by their authors to reduce the contention for channel access in MAC layer and the workload distribution is also better when compared to Single-channel MAC protocols.

VMMAC (VANET Multi-channel MAC): $\mathrm{Xu}$ Xie [3] proposed a directional antenna based adaptive multi-channel MAC protocol for dense VANET. Each vehicle maintains a Beam Table (BT) to show the current state of beams in all seven DSRC channels, the table contains either 1(block) or 0(unblocked) for every beam. Vehicles exchange the BT before sending RTS/CTS handshake for channel reservation. Initially the sender vehicle transmits a Channel Selection Request to Send (CS-RTS) in a predefined direction (assume beam1). After a certain time it transmits the same CS-RTS in all beams around it. The CS-RTS packet contains IDs of sender and receiver, beams of their directional

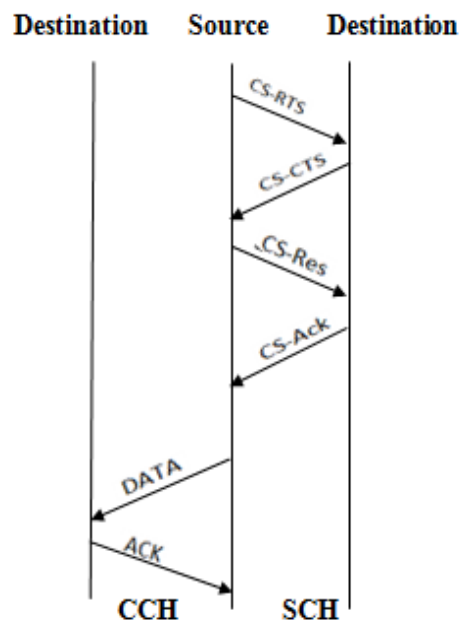

Fig 2: Directional Handshake

antennas, the time duration of the four way handshake and sender BT. Figure 2 describes [3] the directional handshake mechanism in VMMAC. This protocol uses two methods for directional handshake in $\mathrm{CCH}$. In lane-based directional antenna method, CS-RTS packets are transmitted in a circular manner to all beams consecutively. The transmission is changed either when the timer ends or CTS packet is received. This method is suitable in sparse traffic environment with lane limits, such as highway, suburb etc. Omni-directional RTS method solved the problem of contention in dense traffic environment by sending RTS in Omni-directional way.

DMMAC: Ning Lu [8] presented a dedicated multi-channel MAC protocol with adaptive broadcasting mechanism. It is designed to provide collision free and less delay transmissions for safety applications in various traffic environments.

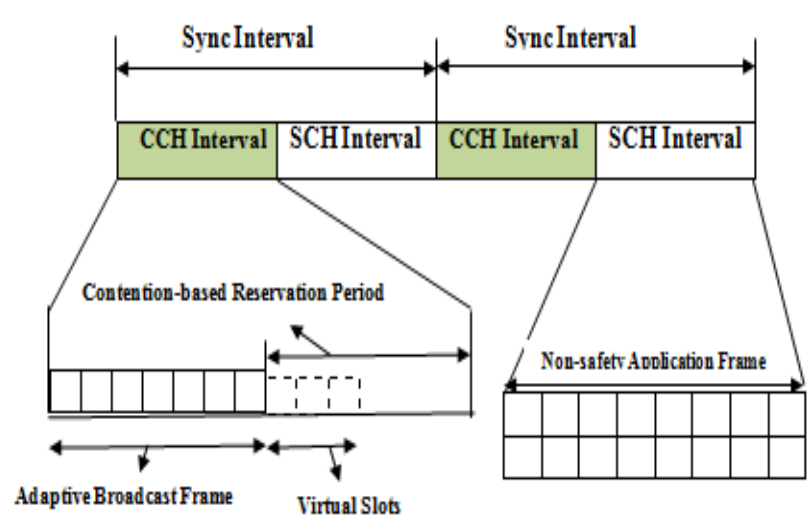

Fig 3: Architecture of DMMAC

The architecture of DMMAC is similar to WAVE MAC which is described in figure 3 . The channel access time is partitioned equally into Sync intervals, and each Sync interval is divided equally into Control channel and Service channel. The Control channel interval is further partitioned into an Adaptive Broadcast Frame (ABF) and a Contention-based Reservation Period (CRP). The Adaptive Broadcast Frame is divided into time slots, and each time slot is reserved by an active vehicle for transmissions of control and safety messages without collisions. The length of the ABF frame is not uniform for entire network. Each vehicle can adjust its ABF length according to its neighbors. The CRP uses CSMA/CA and in CRP vehicles reserve the service channels for non-safety applications. The length of the CRP depends on the $\mathrm{ABF}$ length of the vehicle.

Qing Wang [11] proposed a Quality-of-Service supported multi-channel MAC protocol for VANET which can adjust the size of contention window based on the services at each vehicle, and dynamically adjust the duration of the Control $(\mathrm{CCH})$ and Service Channels (SCHs) working in multi-rate. In this protocol, the Control Channel interval is further divided into safety interval and WAVE Service Announcement (WSA) interval. During safety interval vehicles transmit safety information and broadcast the Variable $\mathrm{CCH}$ Interval (VCI) packets, while in WSA interval service providers broadcast WSA packets with service information and about SCHs. Vehicles can optionally send an acknowledgement (ACK) to the WSA packet. The author has proposed a Markov Chain Model to determine minimum contention window size and $\mathrm{CCH}$ duration for various services.

RAMC: It is a Road Side Unit- Assisted Multi-channel Coordination MAC protocol [1] which utilizes all DSRC channels to provide simultaneous safety and non-safety communications. Vehicles in the transmission range of a roadside unit (RSU) can change to any service channel. The RSU monitors all the safety messages transmitted by both control and service channels and periodically broadcasts a traffic view report to all its neighbor vehicles by using all channels. Vehicles and RSUs use control and service channels in parallel. Time is equally divided, called as sync interval. The length of the interval is measured by the maximum tolerable delay of safety messages. In each sync interval, a vehicle should send at least one safety message by using either control or service channel. So vehicle can operate in a service channel to achieve high throughput for non-safety applications, the author proposed a two-step approach for providing high delivery ratio of safety messages in various 
traffic density conditions. In the first step, a vehicle broadcasts a warning message then the RSU will immediately rebroadcast the message in both control and service channel, while piggybacking acknowledgement (ACK) to the sender vehicle. If the sender does not receive an ACK for a specific amount of time, then it retransmits the message. In the second step, the RSU filters the duplicate messages for the same event.

CMMP: Taeoh Kim [18] proposed a Clustering-Based MultiChannel MAC Protocol for VANET. CMMP separates the channels into control and data channels and figure 4 describes its operation.

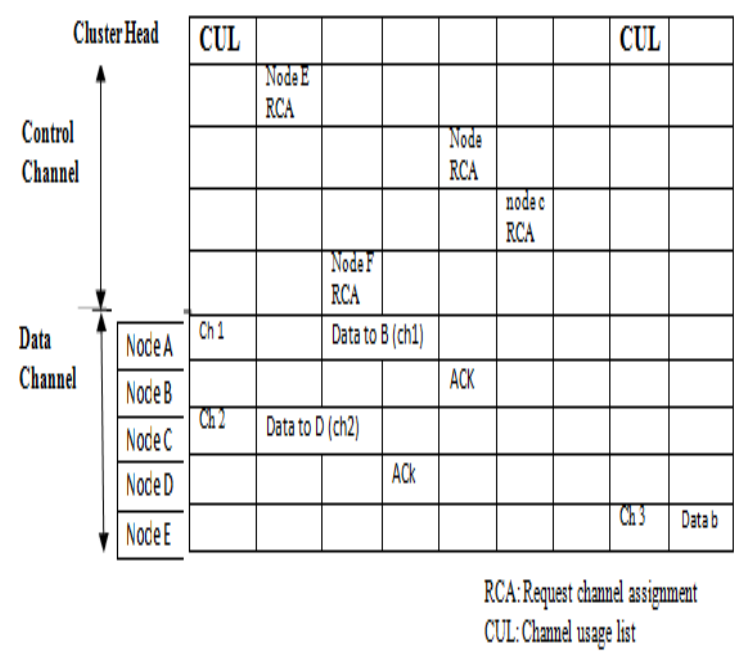

Fig 4: Cluster based Multi-channel MAC Operation

If a cluster member vehicle wants to transmit information to other vehicle then it sends Request Channel Assignment (RCA) packet using CSMA/CA method to the cluster header in order to negotiate the usage of data channel and it will check the Channel Usage List which is periodically broadcasted by the cluster header to know the information about the available channels. If any data channel is available then the vehicle sends its data to the destination, otherwise it retransmits its RCA packet. The channel usage list contains the number of channels, data channel each vehicle is using, and information about the time when the nodes using this channel will change to use other channel. This protocol has decreased transmission delay and packet loss ratio.

Hang Su [15] proposed Clustering-Based Multichannel MAC Protocol for providing quality of service in VANET. This protocol contains three core schemes Cluster Configuration protocol, Intercluster Communication and Intracluster communication protocol. In Cluster Configuration Protocol, vehicles moving in the same direction are grouped into clusters and a vehicle will be elected as a cluster-head. The Intercluster Communication Protocol used to transfer the safety and non safety messages among clusters over two separate IEEE 802.11 MAC-based channels. Intracluster Coordination and Communication Protocol uses multichannel MAC algorithms for each cluster-head to collect/deliver safety messages from/to cluster-member vehicles using the upstream time-division multiple-access/downstream-broadcast method and allocating available data channels to cluster member vehicles for no safety messages.

\section{CONCLUSION}

In this paper we have discussed some of the recently proposed protocols for channel allocation in VANET, where multiple channels are available to transmit the safety and non safety traffic messages among vehicles. The ultimate objective of these protocols is to take the maximum benefit from the available channels in VANET. These protocols have been proved by their authors that contention reduces the performance of single channel MAC layer and the workload distribution is also not well defined. So multi channel MAC protocols are useful to provide better quality of services (QoS). Cluster based multi-channel MAC protocols are improving the performance of overall network in VANET under various traffic environments in safety and non safety applications. In this, multi channel interference is a major problem to assign the channel to a particular vehicle. Therefore cluster based traffic aware channel assignment multi-channel MAC protocol performs well in VANET for various applications.

\section{REFERENCES}

[1] Kai Liu, Jinhua Guo, Ning Lu, Fuqiang Liu, "RAMC: A RSU-Assisted Multi-channel Coordination MAC Protocol for VANET", GLOBECOM Workshops, IEEE 2009.

[2] Yunpeng Zang, Lothar Stibor, Bernhard Walke, HansJürgen Reumerman and Andre Barroso, "A Novel MAC Protocol for Throughput Sensitive Applications in Vehicular Environments", Vehicular Technology Conference, IEEE 2007.

[3] Xu Xie, Benxiong Huang, Shaoshi Yang and Tiejun Lv," Adaptive Multi-channel MAC protocol for dense VANET with Directional Antennas", Future Generation Communication IEEE conference, 2008.

[4] Jonathan Wellons and Yuan Xue," Towards Robust and Efficient Routing in Multi-Radio, Multi-Channel Wireless Mesh Networks”, INFOCOM, IEEE 2011.

[5] Daniel Jiang, Vikas Taliwal, Andreas Meier, And Wieland Holfelder, Ralf Herrtwich And Daimlerchrysler Ag," DESIGN OF 5.9 GHZ DSRC-BASED VEHICULAR SAFETY COMMUNICATION", Wireless Communications, IEEE 2006.

[6] Hamid Menouar and Fethi Filali, Massimiliano, Lenardi and Hitachi," A Survey and Qualitative Analysis of Mac Protocols for Vehicular Ad Hoc Networks", IEEE Wireless Communications, 2006.

[7] Sheng-Hsuan Hsu, Ching-Chi Hsu and Femg-Ching Lin," A Reliable Multi-Channel MAC Protocol with Efficient Multicast Support for Ad hoc Networks", Mobile and Wireless Communication Conference, IEEE,2002.

[8] Ning Lu1, Yusheng Ji, Fuqiang Liu1 and Xinhong Wang," A Dedicated Multi-channel MAC Protocol Design for VANET with Adaptive Broadcasting", Wireless Communications and Networks IEEE Conference, 2010.

[9] Nessrine Chakchouk and Bechir Hamdaoui," Traffic and Interference Aware Scheduling for Multiradio Multichannel Wireless Mesh Networks", IEEE Transactions on Vehicular Technology, Vol 60, No 2, February 2011. 
[10] Tony K Mak, Kenneth P, Laberteaux, Raja Sengupta and Mustafa Ergen," Multichannel Medium Access Control for Dedicated Short-Range Communications", IEEE TRANSACTIONS ON VEHICULAR TECHNOLOGY, VOL 58, NO 1, JANUARY 2009.

[11] Qing Wang, Supeng Leng, Yan Zhang and Huirong Fu," A QoS Supported Multi-channel MAC for Vehicular Ad Hoc Networks", Vehicular Technology Conference, IEEE, 2011.

[12] Tie Luo, Mehul Motani and Vikram Srinivasan," CAMMAC: A Cooperative Asynchronous Multi-Channel MAC Protocol for Ad Hoc Networks", Broadband Communications

Networks, IEEE Conference, 2006.

[13] Zhijun Cai and Mi Lu," SNDR: A New Medium Access Control for Multi-channel Ad Hoc Networks", Vehicular Technology Conference, IEEE, 2000.

[14] Ravi M. Yadumurthy, Adithya Chimalakonda, Mohan Sadashivaiah and Ranga Makanaboyina," Reliable MAC Broadcast Protocol in Directional and Omni-directional Transmissions for Vehicular Ad hoc Networks, 2005 ACM VANET Transaction,2005.

[15] Hang Su and Xi Zhang," Clustering-Based Multichannel MAC Protocols for QoS Provisioning Over Vehicular Ad Hoc Networks", IEEE TRANSACTIONS ON VEHICULAR TECHNOLOGY, VOL 56, NO 6, NOVEMBER 2007.
[16] M. Alicherry, R. Bhatia, and L. Li, "Joint channel assignment and routing for throughput optimization in multi-radio wireless mesh networks," in Proc. of ACM MobiCom, 2005.

[17] Nakjung Choi, Yongho Seok and Yanghee Choi," MultiChannel MAC Protocol for Mobile Ad Hoc Networks", Vehicular Technology Conferencs, IEEE, 2003.

[18] Taeoh Kim, SungDae Jung and SangSun Lee," CMMP: Clustering-Based Multi-Channel MAC Protocol in VANET, Second International Conference on Computer and Electrical Engineering, IEEE, 2009.

[19] Liang Zhou, Baoyu Zheng, Benoit Geller, Anne Wei, Shan Xu , Yajun Li ,"Cross-layer rate control, medium access control and routing design in cooperative VANET”, Elsevier Transaction on Vanet ,2007.

[20] Venkatesh Rajendran, Katia Obraczka and J.J. GarciaLuna-Aceves," DYNAMMA: A DYNAmic Multichannel Medium Access Framework for Wireless Ad Hoc Networks", Mobile Adhoc and Sensor System IEEE Conference, 2007.

[21] Jagadeesh Kakarla, S Siva Sathya, B Govinda Laxmi, Ramesh Babu B,"A Survey on Routing Protocols and its Issues in VANET", International Journal of Computer Applications, Volume 28- No.4, August 2011. 